\title{
COMUNIDAD WAYÚU - EDUCACIÓN Y CULTURA
}

WAYÚU COMMUNITY - EDUCATION AND CULTURE

Resumen

La presente reflexión se enmarca en la Tesis investigativa denominada Cuerpo y Educación en la Cultura Wayúu requisito para optar al título de Magister en Educación, en la Universidad Pedagógica Nacional. Esta investigación se encuentra planteada desde un enfoque etnográfico (Guber, 2001), cualitativo (Coffey \& Atkinson, 2003) e Intercultural; vincula desde el diálogo de saberes, los relatos Wayúu ${ }^{2}$ sobre cuerpo, cultura y educación con el propósito de caracterizar las formas indígenas de estructurar su corporeidad ${ }^{3}$ y la realidad vivida.

No obstante, este artículo aborda específicamente las reflexiones generadas en torno a cultura y educación producto de la tesis investigativa, entendida desde el saber "étnico" o tradición "no occidental".

Palabras clave: corporalidad, educación, cultura Wayúu, educación propia, relatos.

\section{Abstract}

The present reflection places in the research Thesis called Cuerpo y Educación en la Cultura Wayúu requirement to choose to Magister en Educación, at Universidad Pedagógica Nacional. This investigation is raised from an ethnographic approach (Guber, 2001) Qualitative (Coffey \& Atkinson, 2003) Intercultural; it links from the knowledge dialog, the statements Wayúu about body, culture and education with the intention of characterizing the indigenous ways of structuring his bodily and the lived reality.

Key words: bodily, education, Wayúu culture, own education, statements.

Fecha de recepción: 22 de Septiembre de 2010

Fecha de aprobación: 29 de Octubre de 2010

\footnotetext{
${ }^{1}$ Magister en Educación Comunitaria, Especialista en pedagogía, Licenciado en Educación Física. Docente Universidad Pedagógica Nacional, Facultad De Educación Física; Universidad Los Libertadores, Especialización Pedagogía De La Lúdica Para el Desarrollo Cultural; Docente investigador grupo GPAF: Gestión y Pedagogía de la Actividad Física, UPN-Colciencias.

${ }^{2}$ Específicamente la comunidad Wayúu de la ranchería Chispana ubicada en la media Guajira Colombiana, territorio próximo a la ciudad de Riohacha (capital del departamento).

${ }^{3}$ Ó corporalidad, entendido este concepto como el cuerpo construido simbólicamente, según el planteamiento de Jordi Planella, que es subjetivo, sensible, vivencial y experimental, o en términos de Duch ( 2003) "cuando afirmamos que el cuerpo humano es corporeidad queremos señalar que es alguien que posee consciencia de su propia vivacidad, de su presencia aquí y ahora, de su procedencia del pasado y de su orientación al futuro, de sus anhelos de indefinido a pesar de su congénita finitud". 0 entendido desde la perspectiva de expresión simbólica, experiencia a partir y en relación con el cuerpo, como plantea Paredes (1998).
} 


\section{Introducción}

Según Ruano (1994) "los que estudian tienen una historia socio académica determinada, pero los sujetos también cuentan con epistemes que explican su propia realidad" de allí que desde una perspectiva etnográfica, se exploren los relatos cotidianos como propuesta metodológica haciendo posible la construcción de un marco teórico de la comunidad, desde sus actores.

Por otra parte, el presente artículo explora el marco conceptual referente a cultura y educación, que obligatoriamente atraviesa 1 . Los distintos conceptos de cultura que permiten interpretar el contexto de la comunidad Wayúu y 2 . la distinción de tres propuestas socio-políticas de educación como son: la educación formal, la etnoeducación y la educación propia; que entendidas desde la perspectiva cultural difieren en manifestaciones, métodos, tradiciones y propósitos dirigidos a la construcción de sujeto y sociedad, finalizando con algunas hallazgos sobre la relación cuerpo, cultura y educación en la comunidad Wayúu.

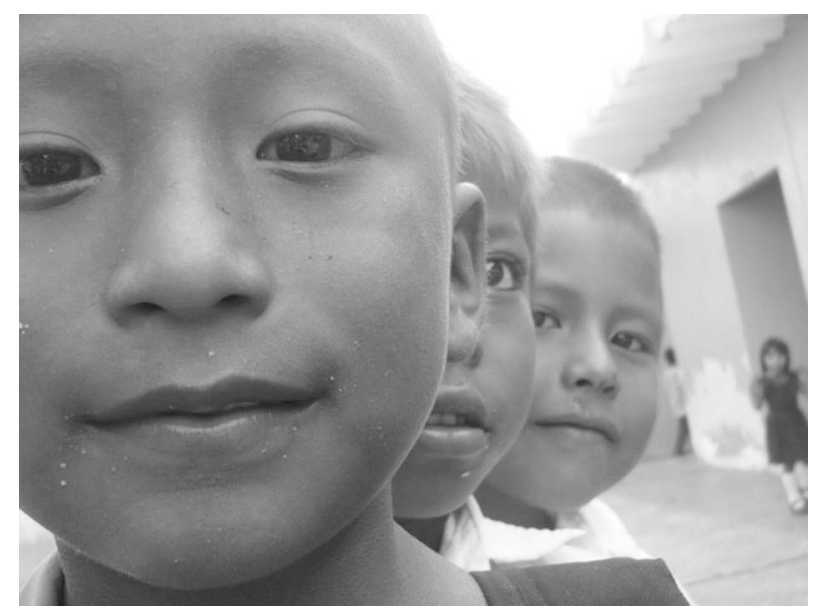

Comunidad Wayúu (Ranchería Chispana) - fotografía de Víctor Hugo Durán Camelo

\section{Metodología}

Se plantea una metodología que retoma aspectos de la investigación de tipo etnográfico, que tiene como principales características: la descripción, la interpretación (hermenéutica) y la comprensión de una realidad a la cual se quiere aproximar, asumiendo algunos aspectos de la metodología de representaciones sociales con respecto a la identificación de las recurrencias en los relatos y el análisis del discur- so cotidiano, ya que la representación, da cuenta de cómo se aprende la cotidianidad y como circula la información desde el contexto particular o de un sujeto a otro (Jodelet, 2000).

\section{Técnicas de Indagación e interpretación}

Las técnicas de indagación responden a las condiciones particulares manifiestas en el contexto de la comunidad Wayúu, determinando para la recolección de datos, la entrevista no directiva, las notas de campo, la grabación in situ, por último, algunos aspectos del análisis del discurso y codificaciones del mismo; abordando como sujetos de la indagación las personas más representativas en la organización social de la comunidad Wayúu (Ranchería Chispana).

\section{Cultura y contexto del pueblo Wayúu}

Para poder entender en qué medida las condiciones y manifestaciones del pueblo Wayúu, se articulan a las prácticas educativas relacionadas con la formación del cuerpo (entendida esta práctica como estructuración de la corporeidad ${ }^{4}$ y a la idea de una forma alternativa de educación, deben explorarse en primer lugar, los conceptos de cultura que circulan cotidianamente y que dan sentido y significado a las prácticas, discursos y simbolizaciones de sus actores, y en segundo lugar, las condiciones particulares socio-económicas, políticas y culturales que distinguen al pueblo Wayúu, entendida como comunidad tradicional (Tönnies, 1986), y que de igual manera fundamentan sus constructos simbólicos.

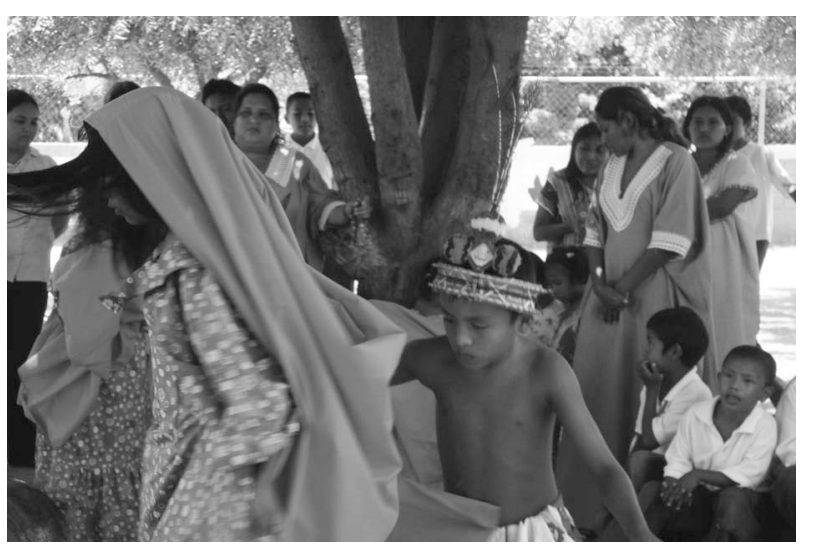

Comunidad Wayúu (Ranchería Chispana) - fotografía de Víctor Hugo Durán Camelo

${ }^{4}$ el cuerpo y sus prácticas son reconocidas como el principal productor simbólico, de subjetividad y fundamento para la construcción de sí mismo e interiorización de la vida social 


\section{Aproximaciones a la cultura}

Observamos el uso cotidiano y exagerado en la utilización del término cultura, en expresiones como: sea culto, iremos al acto cultural, cultura ciudadana, música culta, etc. por lo mismo, se asume bajo un mismo significado, dando por hecho la correcta interpretación del mismo.

Indistintamente hablamos de cultura, cuando se le asemeja al concepto de educación, ilustración, refinamiento, o información vasta; también se le atribuye a la oposición de lo natural, a lo instintivo del hombre. Desde otra perspectiva son las formas que adopta una sociedad unificada por los valores dominantes (Establet, 1966, pp. 12-13), una definición más consensuada, propone la cultura como "el conjunto de los procesos sociales de significación, en donde, "la cultura abarca el conjunto de procesos sociales de producción, circulación y consumo de la significación en la vida social" (García, 2004, p. 32).

Según Malinowski (1994) existen dos axiomas con respecto a cultura 1 . Satisfacción de las necesidades orgánicas en relación con el mundo y su entorno, y 2. Desarrollo de un comportamiento organizado, para poder satisfacer las necesidades del individuo.

Desde el planteamiento de Eco (1998) "toda la cultura, los ritos, las instituciones, las relaciones sociales, las costumbres, etc. No son otra cosa que formas simbólicas en las que el hombre encierra su experiencia para hacerla intercambiable" (Eco, 1998, p. 107), o como lo plantea Geertz (1996). Definiendo la cultura como "urdimbre" en la cual el hombre es un animal inserto en tramas de significación, considerando que el análisis de la cultura no se puede plantear como ciencia experimental "en busca de leyes", sino como ciencia hermenéutica, la cual busca significaciones.

Entre muchos enfoques, este trabajo recoge aquellos conceptos que presentan una relación directa con la significación de lo social, en cuanto a relaciones, producciones, conocimientos y discursos, es decir, la más próxima a las construcciones simbólicas de las comunidades incluyendo entre muchas prácticas aquellas prácticas utilizadas para la educación de la corporalidad.

Por consiguiente, el escenario que exploraremos desde la relación cultura-educación, se encuentra di- rectamente relacionada con aquellas producciones simbólicas que surgen de la cultura "no occidental", articulándose a la educación desde las distintas prácticas, estructuras, discursos e imaginarios, utilizados para favorecer los procesos de enseñanza-aprendizaje en la estructuración de la corporalidad del Wayúu.

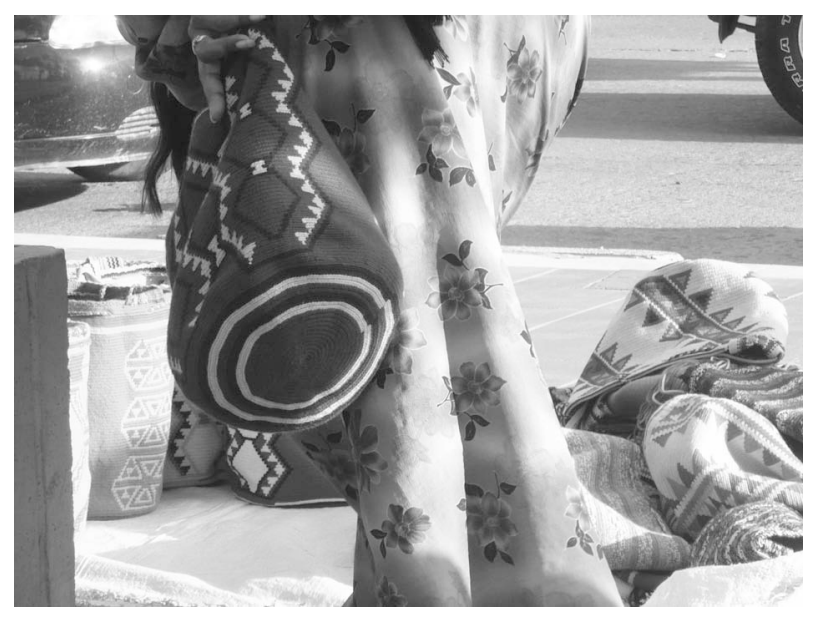

Comunidad Wayúu (Ranchería Chispana) - fotografía de Víctor Hugo Durán Camelo

\section{La Comunidad Wayúu}

La cultura Wayúu según cifras del DANE a partir de los datos arrojados por el censo general del 2005, es la etnia indígena más numeroso de Colombia con una población aproximada de 265.058 integrantes, representando más del $20 \%$ de la población indígena nacional. En Colombia ocupan un área de 1.080.336 hectáreas localizadas en resguardos de la alta y media Guajira, su lengua es el Wayúunaiki, reconocido nacionalmente como "idioma" indígena.

Esta región se caracteriza generalmente por su clima árido, con bajos niveles de lluvia al año, lo que genera como consecuencia que las pocas fuentes de agua que bañan su territorio adquieran una vital importancia en la estructura socio-económica y cultural del Wayúu (Pérez, 1990).

Según sus habitantes, el territorio Wayúu, es actualmente un espacio de gran importancia geopolítica en el caribe colombiano, debido a su gran riqueza en variados yacimientos de recursos naturales, lo cual ha ocasionado diversas problemáticas sociales, económicas y culturales derivadas de las escasas e ineficientes políticas estatales relacionadas con su explotación. 
Pese a la resistencia de los pueblos indígenas, las dinámicas actuales dan cuenta de un proceso sistemático de expropiación y rupturas socio-culturales, utilizando como medio la violencia y el desplazamiento forzado con injerencia de grupos al margen de la ley y empresas multinacionales auspiciadas por el gobierno nacional, según lo indica el documento "para la guajira de las transnacionales y los mega proyectos el pueblo Wayúu sale sobrando"5.

Socialmente presenta una estructura compleja basada en la "matrilinialidad" como sistema de parentesco, es decir, la familia reconocida y el apellido están ligados a la familia materna, conservando aún la estructura de clanes. La comunidad en general tiene la ranchería como modelo para la utilización del espacio, esta manera de poblamiento se manifiesta en una ausencia de centros y periferias y pocos habitantes por $\mathrm{Km}^{2}$.

Dentro de la familia materna, la mayor autoridad le corresponde al tío, dentro de la familia nuclear, los hijos son dirigidos prácticamente por el hermano de la madre y no por el propio padre biológico. Cada ranchería cuenta con su "autoridad tradicional" quien se encarga de dirimir las disputas de la ranchería y organizar la comunidad, en segunda instancia y no menos importante se encuentra al "palabrero" o put'chipu que son los portadores de la palabra y también los que ayudan a resolver conflictos entre los clanes, es el sistema "jurídico" oral de la comunidad, por último se encuentra el/la piache quien representa el vinculo con la dimensión espiritual del Wayúu.

Desde otra perspectiva, un aspecto importante para la comprensión de la comunidad se encuentra en la identificación de los vínculos existentes entre su cosmovisión (que atraviesa las dinámicas sociales-políticas y culturales) y las formas de educación "tradicional" que aún se conservan, es decir, como son transmitidas todas aquellas prácticas, estructuras, discursos e imaginarios relacionados con la constitución de la corporalidad, familia, formas de poblamiento, organización social, sistemas jurídicos, entre otras más particulares como, distribución de

${ }^{5}$ Documento presentado en la sesión del Tribunal Permanente de los Pueblos - Capítulo Colombia, realizada el 18 de julio de 2008 en Atánquez, Resguardo Indígena Kankuamo, Sierra Nevada de Santa Marta) Painwashi, W., \& y Süttsun Jiyeyúu, O. d. (2008). roles de género, sistema espiritual y festivo, vínculos con el territorio, y otros saberes.

Desde esta línea de pensamiento, se hace pertinente revisar desde la perspectiva académica y gubernamental, como ha sido el acercamiento del sistema educativo tradicional "occidental" a estas maneras alternativas de transmitir un saber contextual y de construir una visión de hombre desde el referente "étnico".

\section{Manifestaciones y nuevas perspectivas de educación}

Para Vasco (1985), la educación es un proceso de formación cuando este ocurre en un contexto institucional. Por su parte Lucio, (1990) propone la "educación como un proceso por medio del cual la sociedad facilita de una manera intencional o implícita el crecimiento de sus miembros, es ante todo una práctica social que responde a, o lleva implícita, una determinada visión de hombre" (p. 41).

No obstante, al hablar de educación también debemos entenderla en sus manifestaciones, como dispositivos (Foucault, 1992) para la reproducción cultural, que integra todas aquellas prácticas, saberes, y discursos válidos para preservar en el tiempo una estructura social específica ${ }^{6}$, lo cual no escapa a formas sociales de reproducción e imposición hegemónica.

Desde los planteamientos del Ministerio de Educación Nacional (MEN) en el territorio nacional, la propuesta educativa se encuentra diferenciada en tres modalidades ó categorías: la educación formal ${ }^{7}$, la educación no formal $^{8}$ y la educación informal ${ }^{9}$. Determinando a su vez lineamientos para el desarrollo de sus contenidos, principalmente en las dos primeras, generando unas formas estatales de "edu-

\footnotetext{
${ }^{6}$ Atravesando de manera obligatoria las prácticas corporales como mecanismo de normalización y disciplina miento social y cultural (Foucault, 1976a, p.149).

${ }^{7}$ Cuando está sujeto a las políticas de un sistema educativo que implica una promoción y certificación ya definidas.

${ }^{8}$ Ahora denominado (educación para el trabajo y el desarrollo humano) cuando estos procesos se dan de manera intencional "al margen del sistema educativo formal" (Mariño \& Cendales, 2004) más su intención se centra en la cualificación profesional con proyección laboral.

${ }^{9} \mathrm{La}$ cual hace referencia a todos aquellos aprendizajes que suceden de forma casual en la cotidianidad.
} 
car", que son ratificadas e implementadas a partir de políticas y legislaciones dispuestas para este fin.

Sin embargo, a partir del pronunciamiento de la Constitución Política Colombiana de 1991 el país esboza formas alternativas de pensarse, reconociéndose como nación pluriétnica y multicultural, propiciando nuevos retos y preguntas enfocadas hacia la consolidación de un sistema educativo que promoviera al ser humano desde la diversidad.

Es así como desde los grupos étnicos y el reconocimiento de sus necesidades, expectativas, contexto, cosmovisión y propuestas comunitarias, surgen dinámicas educativas como formas reconocidas de resistencia al sistema de educación tradicional "occidental", como es el caso específico de las propuestas de etnoeducación y educación propia las que parece ser, aún no han sido adecuadamente difundidas.

\section{La Etnoeducación, un intento fallido}

La etnoeducación según Enciso (2004) tiene sus orígenes en Colombia, a partir del año 1985, creada como un programa del Ministerio de Educación Nacional, que en aquel entonces hacia énfasis en tres procesos fundamentales: 1. Capacitación docente, 2. Educación bilingüe y 3. Políticas de acceso de las personas pertenecientes a grupos étnicos, a la educación occidental con base en las políticas de asimilación y descentralización del estado.

Desde la postura docente, esta modalidad generó un enfrentamiento personal en relación con lo pedagógico, a partir de la pregunta sobre qué es lo propio y lo ajeno en los postulados que organizaban la enseñanza para las comunidades indígenas, pues no habían sido formados para hacer esa discriminación de prácticas y saberes, optando en últimas por repetir "los esquemas de su propia experiencia educativa que había sido orientada en todo sentido desde una óptica occidental" (Enciso, 2004, p.12).

Mucho tiempo después iniciativas orientadas por la Organización Nacional Indígena de Colombia (ONIC) en acuerdo con las comunidades negras, propuso al MEN "unos fundamentos y principios que según el criterio de varias organizaciones, habrían de guiar la etnoeducación, [...] convirtiéndose una vez más los requerimientos de los grupos étnicos en política pública" (Enciso, 2004, p.14), basados en criterios como: "territorialidad, cosmovisión, diver- sidad, usos y costumbres y pedagogía. Además de los principios de: integralidad, diversidad lingüística, identidad, autonomía, participación comunitaria, sustentabilidad y viabilidad económica, progresividad, interculturalidad, flexibilidad, cohesión y control social" (Enciso, 2004, p. 14).

Estos fundamentos y principios derivaron en unos acuerdos básicos a la hora de abordar la educación para grupos étnicos, como la concordancia entre las características culturales, las necesidades del grupo étnico y la educación que se debía impartir, el derecho de la comunidad a participar en el diseño de sus programas educativos, la reivindicación de la lengua materna del grupo étnico y la selección de maestros pertenecientes a la misma comunidad (Enciso, 2004, p. 9).

En consecuencia, la etnoeducación se constituyó como un proceso social permanente, inmerso en la cultura propia, que permite, conforme a las necesidades, intereses y aspiraciones de un pueblo, formar a sus individuos para ejercer su capacidad social de decisión, mediante el conocimiento de los recursos de su cultura, teniendo en cuenta los valores del grupo étnico que permitan un reconocimiento y a la vez relación con otras culturas y con la sociedad hegemónica en términos de mutuo respeto (Varios, 2002).

Por otra parte, en el escenario étnico nacional, la noción de etnoeducación generó tensiones en los distintos movimientos sociales, basadas en el evidente distanciamiento de la propuesta pedagógica, articulándose principalmente a las dinámicas sociopolíticas sobre la comprensión de lo étnico.

La percepción de los actores sociales e institucionales acerca de la etnoeducación está profundamente asociada a las ideas que sobre la diferencia cultural y étnica tienen éstos. En gran medida estas concepciones no obedecen a planteamientos de orden educativo, al menos no exclusivamente, sino que expresan definiciones sobre el ser indígena, el ser negro, lo comunitario, la cultura, la tradición, lo étnico, etc. En este sentido, encontramos una noción construida socialmente en el marco de una gran diversidad ideológica y política, desde la cual los diferentes actores sociales organizados tramitan aspiraciones y demandas (Rojas \& Castillo, 2005). 
Crítica que hizo urgente acuñar un nuevo concepto, más próximo a las expectativas particulares, resinificadas a partir del ideario educativo-pedagógico de los colectivos en mención.

En consecuencia, surge la propuesta de "educación propia" como otra forma de resistencia, debido (como ya fue mencionado) a la connotación política e institu cional del término etnoeducación, promulgada junto con sus lineamientos a partir de las políticas estatales, en contraposición, el nuevo planteamiento de la "educación propia" se encuentra sustentada en la formulación de dichos lineamientos a partir de las necesidades y expectativas del propio pueblo.

\section{Educación propia, educar desde la cosmovisión}

En concordancia con la Constitución Política Colombiana de 1991, la Ley General de Educación (Ley 115/94) y la reglamentación del servicio educativo a comunidades indígenas (Decreto 804/95), ratifican las garantías ganadas por las comunidades en décadas anteriores, concretadas en la inclusión de propuestas educativas para grupos étnicos y la política de resguardo y cabildo, entre otras cosas. De igual manera, en 1994 se promulga el Decreto 1860 que reglamenta parcialmente la Ley 115 en sus aspectos pedagógicos y organizativos generales, incluyendo la perspectiva étnica.

Por consiguiente, "la educación propia hace énfasis en aspectos como la integralidad y se complejiza al desbordar el ámbito escolar, ya que residiría fundamentalmente en las dinámicas y manifestaciones culturales particulares de cada pueblo y sus cosmovisiones" (Rojas \& Castillo, 2005).

La educación propia se da en cumplimiento de la Ley de Origen, o derecho mayor, o derecho propio de cada pueblo y mantiene la unidad, la relación con la naturaleza, con otras culturas, con la sociedad mayoritaria y cada una con sus propios usos y costumbres; se desarrolla con base en la sabiduría y conocimiento propio de manera vivencial, con la participación de sabedores ancestrales, autoridades, mayores, padres de familia y comunidad (CONTCEPI, 2008, p. 9).

Lo anterior se basa en el reconocimiento, de que cada pueblo ha construido unas formas particulares de cuerpo, comunicación, trabajo, formación, medicina, espiritualidad, asentamiento en el territorio, convivencia y formas de producción, entre otros, desde la dimensión política, se entiende lo propio como: "la capacidad de orientar, dirigir, organizar y construir los procesos y propuestas educativas con un posicionamiento crítico y propositivo frente a la educación que queremos" (CONTCEPI, 2008).

Por esto, según CONTCEPI (2008) se establecen como principios de la educación propia, la territorialidad como espacio sagrado y posibilidad de vida, la espiritualidad indígena como energía vital y vínculo con lo universal, la familia que se da desde los vínculos de progenitura, pero también desde el parentesco que nos otorga el ser criaturas hijas de la madre tierra familia grande que incluye animales, plantas, lagos, montañas, las lenguas y otras formas de comunicación.

En este sentido, pensar en educación propia en el escenario nacional, es dar cabida a otras formas de manifestación pedagógica, de prácticas, símbolos y construcciones de corporeidad manifiestas, experienciadas y vividas de manera alterna a la propuesta educativa del "ideal" (hegemónico) nacional.

\section{Discusión \\ Educación y cultura, tensiones y hegemonías}

El cuerpo desde la relación entre las distintas culturas, se postula como el más concreto receptáculo de estigmatización y señalamiento de las diferencias, limitaciones, exclusiones e imposición hegemónica, sin embargo, también ha sido desde la corporalidad, fuente de construcción y expresión simbólica, origen, medio y proyección de la experiencia humana que trasciende lo netamente biológico.

En este sentido, la educación del cuerpo ó construcción de la corporalidad es atravesada por estructuras intencionadas de reproducción cultural entre ellas el sistema educativo, el sistema social, y las prácticas culturales, haciéndose fundamental la pregunta, ¿desde dónde hay una intención y dónde se construye dicha corporeidad en los Wayúu?

Como se ha planteado, de un ideal étnico de autoconstrucción, reconocimiento y regulación, surge el concepto de educación propia, pero hasta qué punto estas comunidades dentro de un panorama sociopolítico nacional, tienen la posibilidad de determinar cuáles son los métodos, contenidos, lineamientos y cosmovisiones que guíen la construcción de un ideal de hombre. En otros términos, ¿subsisten aún en los grupos étnicos, formas tradicionales de edu- 
cación (sustentada en la cosmovisión particular), que propicien cuestionamientos sobre: qué, cómo, dónde y para qué se enseña?, ¿qué y desde dónde se evalúa?, ¿qué principios y valores son considerados de importante transmisión?, por último, ¿a qué tipo de ser humano se le apuesta?

A partir de los datos recogidos en la comunidad Wayúu perteneciente a la ranchería Chispana, es evidente en el panorama nacional la hegemonía del sistema educativo tradicional "occidental" (la educación formal), frente a otras formas alternativas de transmisión cultural, generando desde lo formal, críticas y transformaciones directas principalmente en lo concerniente a la corporalidad y sus prácticas consideradas como "educables"; la comisión nacional de trabajo y concertación de la educación para los pueblos indígenas, lo plantean de la siguiente manera:

Nos parece oportuno anotar que esta pérdida, debilitamiento o subordinación de nuestra educación con respecto al modelo educativo hegemónico, ha ido en detrimento no sólo de nosotros mismos sino de la sociedad en general, en tanto que esta situación pasada y presente, ha limitado sustancialmente la oportunidad de compartir y hacer visible el aporte de nuestros saberes y visiones de mundo; que porque no, pueden constituir una alternativa de solución a muchas de las problemáticas de convivencia y permanencia de los seres humanos en esta tierra (CONTCEPI, 2008, p. 14).

Una forma concreta de comprobar esta imposición hegemónica, está dada, en la forma reduccionista de comprender la escuela como sitio socialmente privilegiado para la transmisión de saberes y para el ejercicio de la docencia, desbordando la cultura e institucionalizando las prácticas de la enseñanzaaprendizaje. Las escuelas son espacios nuevos en el territorio Wayúu, allí se lleva al niño como parte de la imposición de la cultura "occidental", para ser disciplinados, normatizados y normalizados (Foucault, 1976), para aprender los otros saberes, la otra cultura, aquella que no les es propia.

Muy distante a lo manifestado por la cultura Wayúu, quienes comprenden la educación como acción permanente, ligado a la vivencia de la cotidianidad, es decir, vivencia, experiencia (corporalidad), diálogo (oralidad), territorio, símbolo, son métodos y espacios de aprendizaje de lo que se considera importante (lo que me sirve para vivir).

Lúdica pedagóg. Vol 2, No. 15 (ene- dic. 2010) pp. 169 - 177
Con ello, se ratifica una dualidad educativa que refuerza los procesos de aculturación en el panorama nacional, en donde, los niños indígenas que en épocas anteriores aprendían de su corporalidad en relación con la naturaleza, de la vida, de su cultura y de sí mismos, en la cotidianidad, con la tutela del adulto desde el vínculo de consanguinidad; hoy en día se enfrentan a la permanencia en un ente externo, extraño y cerrado, como lo es la escuela, en donde es presionado para aprender saberes, prácticas y discursos, que en primer lugar, son abstracciones de la vida real, y en segundo lugar no hacen parte de su cultura.

Por esta razón, como forma de resistencia a la exclusión y la invisibilización, estas posturas alternativas de educación "la educación propia", vienen tomando importancia en el panorama nacional, nos convocan crear desprendimientos de la hegemonía de un saber occidental, para reconocer el potencial presente en otras manifestaciones pedagógicas, epistemológicas y cosmogónicas, vinculadas a los procesos educativos, para:

Garantizar el derecho a la educación propia, para lograr la supervivencia de los pueblos indígenas, el fortalecimiento de la identidad cultural, el desarrollo de proyectos educativos comunitarios, la conservación de las lenguas indígenas y el reconocimiento de las cosmogonías, a partir del dialogo de saberes, la interculturalidad equitativa y la diversidad étnica (CONTCEPI, 2008).

Con respecto a la posibilidad de exaltar nuevos métodos, contenidos y lineamientos educativos relacionados desde esta perspectiva, a la formación, experiencia y expresión de la corporalidad desde la cosmovisión Wayúu como propuesta alternativa.

Sin embargo, existe una crítica que comparten las tres propuestas mencionadas (educación formal, etnoeducación y educación propia), sustentada en la hegemonía cultural, se trata de la obligatoriedad de reproducir el modelo escolar dominante, es decir, no importa si es A ó B, siempre y cuando se desarrollo bajo los parámetros estatales. En este sentido, reiteramos los planteamientos de Foucault (1976a 149), con respecto a la función de la escuela como institución, parte del aparato social para la normalización, normatización y disciplinante del sujeto, incluyendo en este caso la función de aculturación. 


\section{Conclusiones}

Las conclusiones se generan a partir de la triangulación de las fuentes de información, es decir, el marco referencial, las entrevistas no directivas que se realizaron con las personas (roles) más representativos de la comunidad, las notas de campo, la observación directa y las grabaciones insitu, permitiendo evidenciar y priorizar "los relatos" fundamentales de la comunidad.

Por medio de estos relatos se pudo hilar un discurso "cotidiano" compartido por los integrantes de la comunidad, sobre cuerpo, educación y cultura, asignando el debido valor a los saberes otros (Villa y Grueso, 2008) manifiestos; entendidos como parte de ese intercambio simbólico que configura la cultura. Los discursos que se generan y trasmiten a partir de los relatos Wayúu, se integran efectivamente en su cotidianidad para cumplir una doble función, dentro de la actualización y trasmisión cultural, y como referente en los procesos de educación.

Desde esta última perspectiva se muestran dichos relatos como dispositivos que intencionan la experiencia corporal y delimitan su construcción como cuerpos simbólicos, relacionando su condición biológica con el contenido cultural.

En otro sentido, la educación formal (comprendida como sistema educativo) se ha instalado como mecanismo social de reproducción cultural y hegemónicamente ha impuesto unas formas de ser y hacer a partir de las políticas de estado, sin embargo, se reconocen tendencias de resistencia a esta forma única de concebir el ideal de hombre y realidad. Si bien se reconoce que la educación permite moldear las prácticas, los roles y los saberes del niño con el fin de articularlo de manera efectiva a la vida adulta, también se sabe, que es un acto intencional y parcializado, por ende, se encuentra estrechamente ligado a la reproducción de la cosmovisión particular del individuo, mediado por la formación de la corporalidad y sus prácticas.
En este panorama de manifestaciones educativas relacionadas con "las culturas", cada una de ellas adquiere un sentido de importancia y roles que deben cumplir socialmente, por consiguiente, es importante la educación en general porque permite el acceso a la cultura global, y pone a circular los saberes particulares del grupo social en la cual se desarrolla. $\mathrm{z}$

Por su parte, la etnoeducación fue fundamental como un primer intento por reconocer la diversidad cultural en un país como el nuestro, caracterizado por tendencias hegemónicas y homogeneizantes desde la postura occidental, frente al hecho educativo.

En tercer lugar, la educación propia es importante porque trasciende las dos formas anteriores, afianzando la identidad cultural, empoderando a los grupos étnicos con respecto a su desarrollo socioeconómico, político, educativo y cultural, a partir de las características, necesidades y cosmogonías propias, permitiendo a su vez relaciones entre culturas, colocadas en términos de interculturalidad y no de "asimilacionismo" cultural o aculturación.

Como propuesta educativa inmersa en lo cultural (educación propia), se encuentra la tradición Wayúu, en donde los ciclos naturales, desarrollo biológico corporal y cosmovisión, marcan las pauta del aprendizaje; el proceso de enseñanza-aprendizaje va estrechamente ligado a la vida cotidiana desde la oralidad, las prácticas y los discursos (relatos) que se generan; los mayores tiene importancia como sujeto de la experiencia y el saber; y se implementa la educación tradicional desde la autoridad que permite el saber y no la imposición desde los lugares de poder ( jerarquía).

En consecuencia vemos como el Wayúu, culturalmente transita entre la resistencia al mundo occidentalizado-globalizado y la supervivencia, entre la transformación o la desaparición cultural, la defensa del territorio o la expropiación por parte de las multinacionales, a pesar de la dureza del territorio y las precarias condiciones sociales producto del olvido estatal. 


\section{Referencias}

Coffey, A., Atkinson, P. (2003). Encontrar el Sentido a los Datos Cualitativos, Estrategias Complementarias, Medellín, Universidad de Antioquia.

CONTCEPI (2008). 'Perfil del Sistema Educativo Indígena Propio'. En Comisión Nacional de Trabajo y Concertación de la Educación Para los Pueblos Indígenas -CONTCEPI- Subcomisión de Trabajo Para la Construcción, Bogotá, Documento Técnico.

Duch, Li. Y Melich, J. C. (2003). Escenaris de la corporeitat, Barcelona, Publicacions Abadia Montserrat.

Eco, U. (1988). Signo, Barcelona, Labor.

Enciso, P. (2004). Estado del Arte de La Etnoeducación en Colombia con Énfasis en Política Pública, Ministerio de Educación Nacional.

Establet, R. (1966). Culture et idéologie, Paris, Cahiers Marxistes Leninistes.

Foucault, M. (1992a). Microfísica del Poder, Madrid, La piqueta.

Foucault, M. (1976b). Vigilar y castigar, México, Siglo XXI.

García C, N. (2004). Diferentes, Desiguales y Desconectados, Barcelona, Gedisa.

Geertz, C. (1996). Los Usos de la Diversidad. Barcelona, Buenos Aires, México, Paidós.

Guber, R. (2001). La Etnografía, Método, Campo y Reflexividad, Bogotá, Norma.

Jodelet, D., Guerrero, T. (2000). Develando la Cultura, México, Universidad Nacional Autónoma de México.

Lucio, R. (1990). La Construcción del Saber y del Saber Hacer, Bogotá, Tercer Congreso de Educación.

Malinowski, B. (1994). 'The Dynamics of Cultura Change'. En R. Ávila Pedanos, La Educación y el proyecto de la Modernidad, Antropos.

Mariño, G., Cendales, L. (2004). La Educación no Formal y la Educación Popular, Hacia una pedagogía del diálogo cultural, Federación internacional de Fe y Alegría.
Painwashi, W., Süttsun Jiyeyúu, O. (2008). Para La Guajira de las Transnacionales y los Megaproyectos el Pueblo Wayúu Sale Sobrando, Guajira, Atanques.

Paredes, M. (1998). 'La Prueba de Rorschach y la Corporalidad'. En M. L. Rovaletti, Corporalidad, La Problemática del Cuerpo en el Pensamiento Actual, Buenos Aires, Lugar, 371.

Pérez, P. (1990). Evolución Paleo geográfica y Dinámica Actual de los Medios Naturales de la Península de la Guajira, Bogotá, Universidad Nacional.

Rojas, A., Castillo, E. (2005). Educar a los Otros, Estado, políticas educativas y diferencia cultural en Colombia, Cali, Universidad del Cauca.

Ruano, L. (1994). 'De la construcción de los otros por nosotros a la construcción del nosotros'. Obtenido el 30 de Julio de 2009, desde: www.campusmoodle.proed. unc.edu.ar

Tönnies, F. (1986). Revista Sociológica, Teoría Sociológica No.1.

Varios. (2002). Documento Preparatorio III, Encuentro Universitario de Etnoeducación. Universidad Distrital.

Vasco, C. (1985). Práctica Reflexión y Práxis, Colombiana de Educación.

Villa, W., Grueso, A. (2008). Diversidad, Interculturalidad y Construcción de ciudad, Bogotá, Alcaldía Mayor de Bogotá, Universidad Pedagógica Nacional.

\section{Referentes legislativos}

Constitución Política de Colombia (1991).

DANE (2005) Informe censo nacional colombiano.

Ley 115/94 (Ley General de Educación).

Decreto 804/95, (regula la prestación del servicio de educación a grupos indígenas).

Decreto 1860 (reglamenta la Ley 115 con respecto a la educación para grupos étnicos). 